\title{
CUTWIDTH OF ITERATED CATERPILLARS *
}

\author{
LAN LIN ${ }^{1,2}$ AND YIXUN $\operatorname{LiN}^{3}$
}

\begin{abstract}
The cutwidth is an important graph-invariant in circuit layout designs. The cutwidth of a graph $G$ is the minimum value of the maximum number of overlap edges when $G$ is embedded into a line. A caterpillar is a tree which yields a path when all its leaves are removed. An iterated caterpillar is a tree which yields a caterpillar when all its leaves are removed. In this paper we present an exact formula for the cutwidth of the iterated caterpillars.
\end{abstract}

Mathematics Subject Classification. 05C78, 68M10, 68R10.

\section{INTRODUCTION}

The cutwidth problem for graphs, as well as a class of optimal labeling and embedding problems, have significant applications in VLSI designs, network communications and other areas. In particular, the cutwidth is related to a basic parameter, called congestion, in designing microchip circuits (see surveys $[2,4]$ ). Here, a graph $G$ may be thought of as a model of the wiring diagram of an electronic circuit, with the vertices representing components and the edges representing wires connecting them. When a circuit is laid out on a certain architecture (say a path), the maximum number of overlap wires is the congestion, which is one of major parameters determining the electronic performance (the greater the congestion is, the

Keywords and phrases. Circuit layout design, graph labeling, cutwidth, caterpillar, iterated caterpillar.

* Supported by NSFC (11101383, 91218301) and 973 Program of China (2010CB328101).

1 School of Electronics and Information Engineering, Tongji University, Shanghai 200092, China.

2 The Key Laboratory of Embedded System and Service Computing, Ministry of Education, Tongji University, Shanghai 200092, China.

3 Department of Mathematics, Zhengzhou University, Zhengzhou 450052, China.

linyixun@zzu.edu.cn 


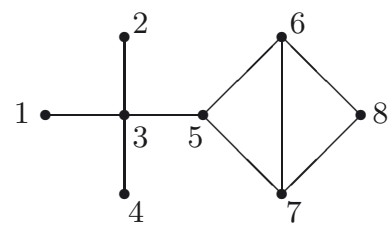

(a) graph $G$ with labels

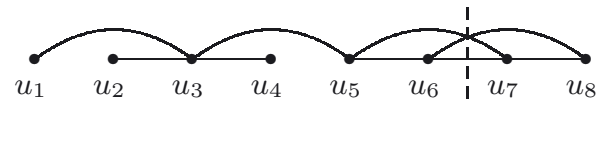

(b) embedding with cutwidth 3

FigurE 1. An example of labeling and embedding.

more interference in the system). This motivates the cutwidth problem in graph theory. In the following discussion, we follow the graph-theoretic terminology and notation of [1].

The problem is formulated as follows. Given a simple graph $G=(V(G), E(G))$ on $n$ vertices, a bijection $f: V(G) \rightarrow\{1,2, \ldots, n\}$ is called a labeling of $G$, which can be thought of as an embedding of $G$ into a path $P_{n}$ of $n$ vertices. For a given labeling $f$ of $G$, the cutwidth of $f$ for $G$ is

$$
c(G, f)=\max _{1 \leq i<n}|\{u v \in E(G): f(u) \leq i<f(v)\}|,
$$

which represents the maximum number of overlap edges (congestion) in the embedding. The cutwidth of $G$ is $c(G)=\min \{c(G, f): f$ is a labeling of $G\}$. A labeling $f$ attaining this minimum is called an optimal labeling.

For example, a graph $G$ is shown in Figure 1a, in which a labeling $f$ is represented by the labels $1,2, \ldots, 8$ besides the vertices. Then the embedding of $G$ on a path is depicted in Figure 1b, in which the maximum number of overlap edges is $c(G, f)=3$.

For a graph $G$, let $S$ be a subset of $V(G)$ and $\bar{S}=V(G) \backslash S$. The edge cut $E[S, \bar{S}]$, i.e., the set of edges of $G$ with one end in $S$ and the other end in $\bar{S}$, is called the coboundary of $S$ and denoted by $\partial(S)$ (following the notation of [1]). For a labeling $f: V(G) \rightarrow\{1,2, \ldots, n\}$ of $G$, let $S_{i}^{f}=\{v \in V(G): f(v) \leq i\}$ denote the set of vertices with the first $i$ labels. Then the above definition is equivalent to

$$
c(G, f)=\max _{1 \leq i<n}\left|\partial\left(S_{i}^{f}\right)\right| .
$$

In other words, let $u_{i}=f^{-1}(i)$ for $1 \leq i \leq n$. Then $S_{i}^{f}=\left\{u_{1}, u_{2}, \ldots, u_{i}\right\}$ and $\partial\left(S_{i}^{f}\right)=\left\{u_{j} u_{k} \in E(G): j \leq i<k\right\}$. The cutwidth $c(G, f)$ is the maximum size of these coboundaries $\partial\left(S_{i}^{f}\right)$. When there is no confusion, we may abbreviate $S_{i}^{f}$ to $S_{i}$ as $f$ is known. In Figure 1, we have $c(G, f)=\left|\partial\left(S_{6}^{f}\right)\right|=3$.

As immediate consequences of the definition, we have the following basic properties of the cutwidth (for the former see Lem. 3 of [3]).

Lemma 1.1. If $G^{\prime}$ is a subdivision of $G$ (that is, some edges are replaced by paths), then $c\left(G^{\prime}\right)=c(G)$. 


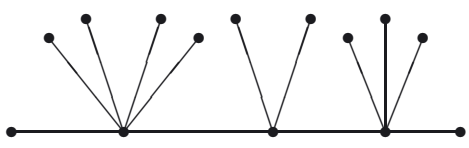

(a)

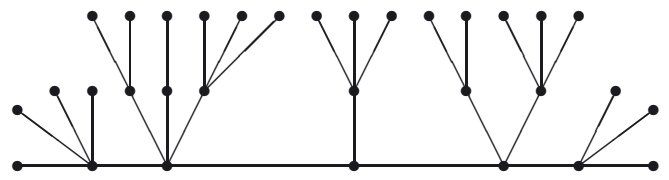

(b)

Figure 2. Caterpillar and iterated caterpillar.

Lemma 1.2. If $G^{\prime}$ is a subgraph of $G$, then $c\left(G^{\prime}\right) \leq c(G)$.

The cutwidth problem for a general graph is known to be NP-complete [6], and it admits a polynomial algorithm for trees [15]. Much work has been done for determining the exact value of the cutwidth of special classes of graphs (see, e.g., $[4,7-10,12,14])$.

In this paper we study basic classes of trees, the caterpillars and iterated caterpillars, providing an exact formula to compute them. A caterpillar is a tree which yields a path when all its leaves (vertices of degree one) are removed, and an iterated caterpillar is a tree which yields a caterpillar when all its leaves are removed (see Fig. 2). In the study of graceful labeling problem [5], the iterated caterpillars are also called lobsters.

The paper is organized as follows. In Section 2, we describe a decomposition approach for caterpillars and iterated caterpillars. Sections 3 and 4 are devoted to the main results on the iterated caterpillars, the lower bound and the exact formula. A summary with connection to the bandwidth problem is given in Section 5 .

\section{Decomposition Approach}

Now we consider a caterpillar $T$, in which the path obtained from $T$ by deleting all leaves is called the backbone of $T$, denoted $p(T)=\left(w_{1}, w_{2}, \ldots, w_{m}\right)$. Let $n_{i}$ be the number of neighbors of $w_{i}(i=1,2, \ldots, m)$. Then $T$ is formed by $m$ stars $T_{1}=K_{1, n_{1}}, T_{2}=K_{1, n_{2}}, \ldots, T_{m}=K_{1, n_{m}}$ with $n=|V(T)|=n_{1}+n_{2}+\ldots+n_{m}-$ $m+2$. That is, $T=\cup_{i=1}^{m} T_{i}$ with the edge $w_{i} w_{i+1}$ belonging to both $T_{i}$ and $T_{i+1}$ $(1 \leq i \leq m-1)$.

For a set $A=\left\{a_{1}, a_{2}, \ldots, a_{k}\right\}$ of $k$ distinct numbers with $a_{1}<a_{2}<\ldots<a_{k}$, if $k$ is odd, the number $a_{\lceil k / 2\rceil}$ is called the median of $A$; if $k$ is even, the numbers $a_{k / 2}$ and $a_{k / 2+1}$ are called the medians of $A$.

A crucial fact is that the cutwidth problem of caterpillar $T$ can be decomposed into $m$ subproblems for each star $T_{i}$. By the center of a star $K_{1, n}$, we mean the vertex adjacent to all other vertices. We begin with an obvious fact for stars as follows ( $c f .[2]$ among others).

Proposition 2.1. Let $T=K_{1, n}$ be a star with center $w$. Then $c(T)=\lceil n / 2\rceil$ and $f$ is an optimal labeling if and only if $f(w)$ is a median of the label set $\{f(v): v \in$ $V(T)\}$. 
Proof. Suppose that $f(w)=i+1$ for an arbitrary labeling $f$. Then $\left|\partial\left(S_{i}\right)\right|=i$ and $\left|\partial\left(S_{i+1}\right)\right|=n-i$. Thus $c(T, f) \geq \max \{i, n-i\} \geq\lceil n / 2\rceil$. On the other hand, we define a labeling $f^{*}$ such that $f^{*}(w)=\lfloor n / 2\rfloor+1$ is a median of the label set. Then $c\left(T, f^{*}\right)=\max \{\lfloor n / 2\rfloor,\lceil n / 2\rceil\}=\lceil n / 2\rceil$ attaining the above lower bound. Thus $f^{*}$ is an optimal labeling and $c(T)=c\left(T, f^{*}\right)=\lceil n / 2\rceil$.

Conversely, if $f(w)=i+1$ is not a median of $\{f(v): v \in V(T)\}$ for some labeling $f$, then $c(T, f) \geq \max \{i, n-i\}>\lceil n / 2\rceil$, and so $f$ is not optimal. This completes the proof.

We further obtain the following.

Proposition 2.2. Let $T=\cup_{i=1}^{m} T_{i}$ be a caterpillar with $T_{i}=K_{1, n_{i}} \quad(i=$ $1,2, \ldots, m)$. Then

$$
c(T)=\max _{1 \leq i \leq m} c\left(T_{i}\right)=\max _{1 \leq i \leq m}\left\lceil n_{i} / 2\right\rceil .
$$

Proof. Since $T_{i}$ is a subgraph of $T$, we have $c\left(T_{i}\right) \leq c(T)$ by Lemma 1.2. Hence we obtain the lower bound $c(T) \geq \max _{1 \leq i \leq m} c\left(T_{i}\right)$. On the other hand, we define a labeling $f^{*}$ as follows: The stars $T_{1}, T_{2}, \ldots, T_{m}$ are labeled in turn by the numbers $\{1,2, \ldots, n\}$ (where $n=n_{1}+n_{2}+\ldots n_{m}-m+2$ ) such that $f^{*}\left(w_{i}\right)$ is a median of $\left\{f^{*}(v): v \in V\left(T_{i}\right)\right\} \quad(1 \leq i \leq m)$. Then this labeling $f^{*}$ restricted to each subtree $T_{i}$ is an optimal labeling of $T_{i}$. Consequently, $c\left(T, f^{*}\right)=\max _{1 \leq i \leq m} c\left(T_{i}\right)=\max _{1 \leq i \leq m}\left\lceil n_{i} / 2\right\rceil$, whence $f^{*}$ is an optimal labeling of $T$. The proof is complete.

We proceed to consider an iterated caterpillar T. A hair is an edge incident with a leaf. Meanwhile, the neighbor set of vertex $v$ is denoted by $N(v)=\{u \in V(T)$ : $u v \in E(T)\}$. Suppose that when deleting all leaves, $T$ becomes a caterpillar $T^{\prime}$ with backbone $\left(w_{1}, w_{2}, \ldots, w_{m}\right)$. Then we define $p(T)=\left(w_{1}, w_{2}, \ldots, w_{m}\right)$ to be the backbone of iterated caterpillar $T$. Moreover, assume that no hair is directly incident with $w_{i}$ for $1 \leq i \leq m$, for otherwise we may subdivide this hair by inserting a new vertex (see Lem. 1.1).

Now we decompose $T$ into subtrees $T_{1}, T_{2}, \ldots, T_{m}$ as follows. For $1 \leq i \leq m$, let $L_{i}$ be the set of leaves of $T$ which are adjacent to vertices in $N\left(w_{i}\right)$. Then $T_{i}$ is a tree induced by $\left\{w_{i}\right\} \cup N\left(w_{i}\right) \cup L_{i}$. Note that $w_{i} w_{i+1}$ is contained in both $T_{i}$ and $T_{i+1}(1 \leq i \leq m-1)$. These $T_{i}$ 's, $1 \leq i \leq m$, are called iterated stars, each of which yields a star when the leaves are removed. Besides, each of $T_{i}$ 's is called a section of $T$. Among them, $T_{1}$ and $T_{m}$ are called end sections and $T_{i}(1<i<m)$ are intermediate sections. This decomposition is shown in Figure 3. Here, we make a convention that the edge $w_{i} w_{i+1}$ belongs to both $T_{i}$ and $T_{i+1}$ (which is ambiguous in Fig. 3).

By virtue of Lemma 1.2 we obtain the following lower bound.

Proposition 2.3. Let $T=\cup_{i=1}^{m} T_{i}$ be an iterated caterpillar with $T_{i}$ being iterated stars $(i=1,2, \ldots, m)$. Then

$$
c(T) \geq \max _{1 \leq i \leq m} c\left(T_{i}\right) .
$$




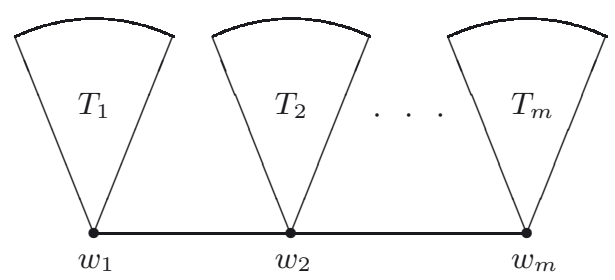

Figure 3. Decomposition of iterated caterpillar.

In addition to Lemma 1.1, another important feature of the cutwidth problem is the following property due to Chung [3].

Lemma 2.4 ([3]). For the cutwidth problem of a tree $T$, there exists an optimal labeling $f$ satisfying the following properties:

(1) The leaf property: the vertices labeled by 1 and $n$ are leaves of $T$.

(2) The monotone property: Let $P=\left(v_{0}, v_{1}, \ldots, v_{l}\right)$ be the path connecting the vertices labeled by 1 and $n$, where $f\left(v_{0}\right)=1, f\left(v_{l}\right)=n$. Then $f\left(v_{0}\right)<f\left(v_{1}\right)<$ $\ldots<f\left(v_{l}\right)$.

(3) The block property: Let $F$ be the forest obtained from $T$ by removing the edges of $P$, where $P=\left(v_{0}, v_{1}, \ldots, v_{l}\right)$ is defined in (2). Then any connected component (maximal subtree) of $F$ is labeled by a set of consecutive integers.

We apply this result to an iterated caterpillar $T=\cup_{i=1}^{m} T_{i}$. Let $V_{1}=V\left(T_{1}\right) \backslash$ $\left\{w_{2}\right\}, V_{m}=V\left(T_{m}\right) \backslash\left\{w_{m-1}\right\}$, and $V_{i}=V\left(T_{i}\right) \backslash\left\{w_{i-1}, w_{i+1}\right\}(1<i<m)$. Then $\left(V_{1}, V_{2}, \ldots, V_{m}\right)$ forms a partition of $V(T)$. We can further show that there exists an optimal labeling $f$ such that $f^{-1}(1) \in V_{1}$ and $f^{-1}(n) \in V_{m}$, and so the path $P$ connecting these two vertices passes through $w_{1}, w_{2}, \ldots, w_{m}$. Therefore we have the following consequence.

Corollary 2.5. For any iterated caterpillar $T=\cup_{i=1}^{m} T_{i}$, there exists an optimal labeling $f$ satisfying the following properties:

(i) $f\left(w_{1}\right)<f\left(w_{2}\right)<\ldots<f\left(w_{m}\right)$.

(ii) Each subset $V_{i}(1 \leq i \leq m)$ is labeled by a set of consecutive integers.

For convenience, this labeling $f$ is called a proper labeling. Henceforth, we can only consider proper labelings for any iterated caterpillar $T$.

\section{LOWER BOUND}

By Proposition 2.3, in order to derive lower bounds for the cutwidth of iterated caterpillar $T=\cup_{i=1}^{m} T_{i}$, we need only consider each section $T_{i}$ for $1 \leq i \leq m$.

We first consider an intermediate section $T_{i}(1<i<m)$. As mentioned before, $T_{i}$ is an iterated star induced by $\left\{w_{i}\right\} \cup N\left(w_{i}\right) \cup L_{i}$, which contains two special 


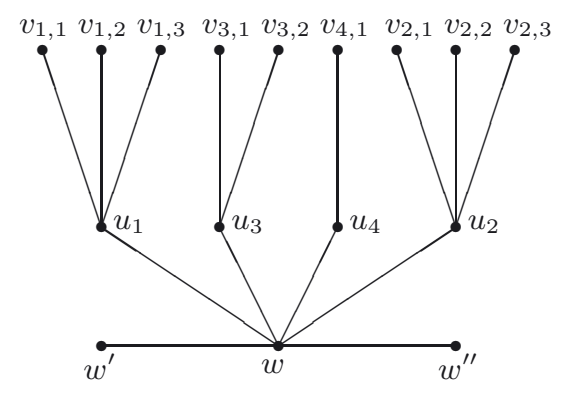

FigurE 4. An intermediate section $T(4,4,3,2)$.

leaves $w_{i-1}, w_{i+1} \in N\left(w_{i}\right)$. We may call $w_{i}$ the root of $T_{i}$ and $w_{i-1}, w_{i+1}$ the backbone leaves.

In general, we define an iterated star $T_{S}=T\left(n_{1}, n_{2}, \ldots, n_{k}\right)$ with root $w$ as follows. Let $T^{1}=K_{1, n_{1}}, T^{2}=K_{1, n_{2}}, \ldots, T^{k}=K_{1, n_{k}}$ be $k$ stars, where $n_{i} \geq 2, i=$ $1,2, \ldots, k$. Then we take one leaf from each star $T^{j}$ and merge these $k$ vertices into a single vertex $w$. In addition, $w$ has two special neighbors $w^{\prime}$ and $w^{\prime \prime}$, which are backbone leaves. Let $u_{j}$ denote the center of star $T^{j}$ and let $v_{j, 1}, v_{j, 2}, \ldots, v_{j, n_{j}-1}$ denote the leaves (other than $w$ ) of $\operatorname{star} T^{j}(j=1,2, \ldots, k)$. An example is shown in Figure 4.

By virtue of Corollary 2.5, for an iterated caterpillar $T$, we always assume that the considered labeling $f$ is proper. So we have $f\left(w^{\prime}\right)<f(w)<f\left(w^{\prime \prime}\right)$ in $T_{S}$. Moreover, we assume that $f\left(w^{\prime}\right)$ and $f\left(w^{\prime \prime}\right)$ are given when discussing the labeling $f$ in $T^{1} \cup T^{2} \cup \ldots \cup T^{k}$ (as $w^{\prime}$ and $w^{\prime \prime}$ are roots of other iterated stars). To derive the lower bound, we need the following sequencing property.

Proposition 3.1. Let $\left(a_{1}, a_{2}, \ldots, a_{k}\right)$ and $\left(b_{1}, b_{2}, \ldots, b_{k}\right)$ be two sequences of real numbers where $a_{1} \leq a_{2} \leq \ldots \leq a_{k}$. Then among all permutations $\pi$ of $\{1,2, \ldots, k\}$, the value $\max _{1 \leq i \leq k}\left(a_{i}+b_{\pi(i)}\right)$ is minimum if $b_{\pi(1)} \geq b_{\pi(2)} \geq \ldots \geq$ $b_{\pi(k)}$.

Proof. We define a function $g(\pi)=\max _{1 \leq i \leq k}\left(a_{i}+b_{\pi(i)}\right)$ on the set of all permutations $\pi$. A permutation $\pi$ is said to be optimal if $g(\pi)$ is minimum. Now we proceed to show that $\pi$ is optimal if $b_{\pi(1)} \geq b_{\pi(2)} \geq \ldots \geq b_{\pi(k)}$. Suppose that there is an optimal permutation $\pi$ such that $b_{\pi(i)}<b_{\pi(i+1)}$ for some index $i$. Then we can construct another permutation $\pi^{\prime}$ by exchanging the positions $\pi(i)$ and $\pi(i+1)$, that is, $\pi^{\prime}(i)=\pi(i+1), \pi^{\prime}(i+1)=\pi(i)$ and $\pi^{\prime}(j)=\pi(j)$ for $j \neq i, i+1$. It follows from $a_{i} \leq a_{i+1}$ and $b_{\pi(i)}<b_{\pi(i+1)}$ that

$$
\begin{array}{r}
\max \left\{a_{i}+b_{\pi(i)}, a_{i+1}+b_{\pi(i+1)}\right\}=a_{i+1}+b_{\pi(i+1)} \geq \max \left\{a_{i}+b_{\pi(i+1)}, a_{i+1}+b_{\pi(i)}\right\} \\
=\max \left\{a_{i}+b_{\pi^{\prime}(i)}, a_{i+1}+b_{\pi^{\prime}(i+1)}\right\}
\end{array}
$$


Let $R=\max \left\{a_{j}+b_{\pi(j)}: 1 \leq j \leq k, j \neq i, i+1\right\}$. Then $g(\pi)=\max \left\{R, a_{i}+\right.$ $\left.b_{\pi(i)}, a_{i+1}+b_{\pi(i+1)}\right\} \geq \max \left\{R, a_{i}+b_{\pi^{\prime}(i)}, a_{i+1}+b_{\pi^{\prime}(i+1)}\right\}=g\left(\pi^{\prime}\right)$. Thus $\pi^{\prime}$ is also optimal. If this $\pi^{\prime}$ does not satisfy the condition $b_{\pi^{\prime}(1)} \geq b_{\pi^{\prime}(2)} \geq \ldots \geq b_{\pi^{\prime}(k)}$, then we carry out the same transformation. In this way, we can eventually obtain an optimal permutation satisfying the required condition. However, all permutations satisfying this condition have the same value of $g(\pi)$. Therefore, every permutation satisfying $b_{\pi(1)} \geq b_{\pi(2)} \geq \ldots \geq b_{\pi(k)}$ is optimal. This completes the proof.

This sequencing property is similar to the well-known property for $\sum_{1 \leq i \leq k} a_{i} b_{\pi(i)}$, only the addition "+" is replaced by "max" and the multiple " $x "$ is replaced by "+".

Proposition 3.2. Suppose that an intermediate section $T_{i}$ is an iterated star $T_{S}=$ $T\left(n_{1}, n_{2}, \ldots, n_{k}\right)$. Then for any proper labeling $f$, a lower bound of the cutwidth is given by

$$
c\left(T_{S}, f\right) \geq \max 1 \leq j \leq k\left(\lceil j / 2\rceil+\left\lceil n_{j} / 2\right\rceil\right),
$$

where $n_{1} \geq n_{2} \geq \ldots \geq n_{k}$.

Proof. Given an iterated star $T_{S}=T\left(n_{1}, n_{2}, \ldots, n_{k}\right)$, the edges are divided into two parts: one part consists of those edges between $w$ and $u \in N(w)$ (including $\left.w w^{\prime}, w w^{\prime \prime}\right)$; the other part consists of those hairs between $u_{j}$ and $v_{j, l}$ for $1 \leq j \leq$ $k, 1 \leq l \leq n_{j}-1$. Let $f$ be a proper labeling of $T_{S}$.

We consider a vertex $u_{j}$ with $f\left(u_{j}\right)<f(w)(1 \leq j \leq k)$. Suppose that $A_{j}=$ $\left\{u w \in E\left(T_{S}\right): u \in N(w), f(u)<f\left(u_{j}\right)\right\}$ (including $w^{\prime} w$ ) and $a_{j}=\left|A_{j}\right| \geq 1$. Meanwhile, suppose that $B_{j}=\left\{u_{j} v_{j, l} \in E\left(T_{S}\right): v_{j, l} \in N\left(u_{j}\right), f\left(v_{j, l}\right)<f\left(u_{j}\right)\right\}$. Then $A_{j} \cup B_{j} \subseteq \partial\left(S_{f\left(u_{j}\right)-1}\right)$. Thus $\left|\partial\left(S_{f\left(u_{j}\right)-1}\right)\right| \geq\left|A_{j}\right|+\left|B_{j}\right|$. On the other hand, let $\bar{B}_{j}=\left\{u_{j} v_{j, l} \in E\left(T_{S}\right): v_{j, l} \in N\left(u_{j}\right), f\left(v_{j, l}\right)>f\left(u_{j}\right)\right\} \cup\left\{u_{j} w\right\}$. Then $A_{j} \cup \bar{B}_{j} \subseteq$ $\partial\left(S_{f\left(u_{j}\right)}\right)$. Thus $\left|\partial\left(S_{f\left(u_{j}\right)}\right)\right| \geq\left|A_{j}\right|+\left|\bar{B}_{j}\right|$. Combining these two inequalities and noting $B_{j} \cup \bar{B}_{j}=E\left(K_{1, n_{j}}\right)$, we have

$$
\begin{aligned}
c\left(T_{S}, f\right) & \geq \max \left\{\left|\partial\left(S_{f\left(u_{j}\right)-1}\right)\right|,\left|\partial\left(S_{f\left(u_{j}\right)}\right)\right|\right\} \\
& \geq \max \left\{\left|A_{j}\right|+\left|B_{j}\right|,\left|A_{j}\right|+\left|\bar{B}_{j}\right|\right\} \geq a_{j}+\left\lceil n_{j} / 2\right\rceil .
\end{aligned}
$$

Symmetrically, for a vertex $u_{j}$ with $f\left(u_{j}\right)>f(w)(1 \leq j \leq k)$, suppose that $A_{j}^{\prime}=\left\{w u \in E\left(T_{S}\right): u \in N(w), f(u)>f\left(u_{j}\right)\right\}$ (including $w w^{\prime \prime}$ ) and $a_{j}^{\prime}=\left|A_{j}^{\prime}\right| \geq 1$. Meanwhile, suppose that $B_{j}^{\prime}=\left\{u_{j} v_{j, l} \in E\left(T_{S}\right): v_{j, l} \in N\left(u_{j}\right), f\left(v_{j, l}\right)>f\left(u_{j}\right)\right\}$. Then $A_{j}^{\prime} \cup B_{j}^{\prime} \subseteq \partial\left(S_{f\left(u_{j}\right)}\right)$. Thus $\left|\partial\left(S_{f\left(u_{j}\right)}\right)\right| \geq\left|A_{j}^{\prime}\right|+\left|B_{j}^{\prime}\right|$. On the other hand, let $\bar{B}_{j}^{\prime}=\left\{u_{j} v_{j, l} \in E\left(T_{S}\right): v_{j, l} \in N\left(u_{j}\right), f\left(v_{j, l}\right)<f\left(u_{j}\right)\right\} \cup\left\{u_{j} w\right\}$. Then $A_{j}^{\prime} \cup \bar{B}_{j}^{\prime} \subseteq$ $\partial\left(S_{f\left(u_{j}\right)-1}\right)$. Thus $\left|\partial\left(S_{f\left(u_{j}\right)-1}\right)\right| \geq\left|A_{j}^{\prime}\right|+\left|\bar{B}_{j}^{\prime}\right|$. Combining these two inequalities and noting $B_{j}^{\prime} \cup \bar{B}_{j}^{\prime}=E\left(K_{1, n_{j}}\right)$, we have

$$
\begin{aligned}
c\left(T_{S}, f\right) & \geq \max \left\{\left|\partial\left(S_{f\left(u_{j}\right)-1}\right)\right|,\left|\partial\left(S_{f\left(u_{j}\right)}\right)\right|\right\} \\
& \geq \max \left\{\left|A_{j}^{\prime}\right|+\left|B_{j}^{\prime}\right|,\left|A_{j}^{\prime}\right|+\left|\bar{B}_{j}^{\prime}\right|\right\} \geq a_{j}^{\prime}+\left\lceil n_{j} / 2\right\rceil .
\end{aligned}
$$




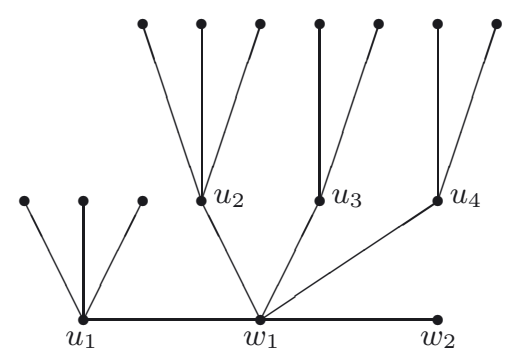

Figure 5. An end section $T^{\prime}(4,4,3,3)$.

To unify the notation, we also denote $a_{j}^{\prime}$ by $a_{j}$ depending on either $f\left(u_{j}\right)<f(w)$ or $f\left(u_{j}\right)>f(w)$. In summary, we obtain the following lower bound:

$$
c\left(T_{S}, f\right) \geq \max _{1 \leq j \leq k}\left(a_{j}+\left\lceil n_{j} / 2\right\rceil\right) .
$$

In this context, for all vertices $u_{j}$ with $1 \leq j \leq k$, the sequence of numbers $a_{j}$ is $(1,1,2,2, \ldots, k / 2, k / 2)$ (if $k$ is even) or $(1,1,2,2, \ldots,(k-1) / 2,(k-1) / 2,(k+1) / 2)$ (if $k$ is odd). This sequence can be written as $(\lceil j / 2\rceil: j=1,2, \ldots, k)$. Note that this sequence $a_{j}=\lceil j / 2\rceil$ is monotonically increasing. By Proposition 3.1, the above lower bound attains its minimum value when $b_{j}=\left\lceil n_{j} / 2\right\rceil$ is monotonically decreasing. As a result, we obtain

$$
c\left(T_{S}, f\right) \geq \max _{1 \leq j \leq k}\left(\lceil j / 2\rceil+\left\lceil n_{j} / 2\right\rceil\right),
$$

where $n_{1} \geq n_{2} \geq \ldots \geq n_{k}$. Thus the assertion is proved.

We next consider an end section $T_{1}$. This subtree $T_{1}$ is induced by $\left\{w_{1}\right\} \cup$ $N\left(w_{1}\right) \cup L_{1}$, which contains only one special leaf $w_{2} \in N\left(w_{1}\right)$, the backbone leaf. As above, we can define an iterated star $T_{S}^{\prime}=T^{\prime}\left(n_{1}, n_{2}, \ldots, n_{k}\right)=K_{1, n_{1}} \cup K_{1, n_{2}} \cup$ $\ldots \cup K_{1, n_{k}} \cup\left\{w_{1} w_{2}\right\}$ with the root $w_{1}$ and only one backbone leaf $w_{2}$. An example is shown in Figure 5.

Proposition 3.3. Suppose that the end section $T_{1}$ is an iterated star $T_{S}^{\prime}=$ $T^{\prime}\left(n_{1}, n_{2}, \ldots, n_{k}\right)$. Then for any proper labeling $f$, a lower bound of the cutwidth is given by

$$
c\left(T_{S}^{\prime}, f\right) \geq \max _{1 \leq j \leq k}\left(\lceil(j-1) / 2\rceil+\left\lceil n_{j} / 2\right\rceil\right),
$$

where $n_{1} \geq n_{2} \geq \ldots \geq n_{k}$.

Proof. As in Proposition 3.2, the iterated star $T_{S}^{\prime}=T^{\prime}\left(n_{1}, n_{2}, \ldots, n_{k}\right)$ has two parts of edges: those edges between $w_{1}$ and $u \in N\left(w_{1}\right)$ (including $w_{1} w_{2}$ ) and those hairs between $u_{j}$ and $v_{j, l}\left(1 \leq l \leq n_{j}-1\right)$. 
For a vertex $u_{j}$ with $f\left(u_{j}\right)<f\left(w_{1}\right)$, define $A_{j}=\left\{u w \in E\left(T_{S}^{\prime}\right): u \in\right.$ $\left.N\left(w_{1}\right), f(u)<f\left(u_{j}\right)\right\}$ and $a_{j}=\left|A_{j}\right|$. Note that there is no $w^{\prime} w_{1}$ with $f\left(w^{\prime}\right)<$ $f\left(w_{1}\right)$ in $A_{j}$ now. So it is possible that $a_{j}=0$ when $f\left(u_{j}\right)$ is minimal. By the same argument as in Proposition 3.2, we can deduce the lower bound

$$
c\left(T_{S}^{\prime}, f\right) \geq a_{j}+\left\lceil n_{j} / 2\right\rceil .
$$

Symmetrically, for a vertex $u_{j}$ with $f\left(u_{j}\right)>f(w)$, we define $A_{j}^{\prime}=\left\{w u \in E\left(T_{S}\right)\right.$ : $\left.u \in N(w), f(u)>f\left(u_{j}\right)\right\}$ (including $w_{1} w_{2}$ ) and $a_{j}^{\prime}=\left|A_{j}^{\prime}\right| \geq 1$. A similar argument shows that

$$
c\left(T_{S}^{\prime}, f\right) \geq a_{j}^{\prime}+\left\lceil n_{j} / 2\right\rceil .
$$

By unifying $a_{j}^{\prime}$ and $a_{j}$, we obtain:

$$
c\left(T_{S}^{\prime}, f\right) \geq \max { }_{1 \leq j \leq k}\left(a_{j}+\left\lceil n_{j} / 2\right\rceil\right) .
$$

For all vertices $u_{j}$ with $1 \leq j \leq k$, the sequence of numbers $a_{j}$ is $(0,1,1,2,2, \ldots,(k-1) / 2,(k-1) / 2)$ (if $k$ is odd) or $(0,1,1,2,2, \ldots, k / 2-1, k / 2-$ $1, k / 2)$ (if $k$ is even). This sequence can be written as $(\lceil(j-1) / 2\rceil: j=1,2, \ldots, k)$. By Proposition 3.1, the above lower bound attains its minimum value when $\left\lceil n_{j} / 2\right\rceil$ is monotonically decreasing. Hence we obtain

$$
c\left(T_{S}^{\prime}, f\right) \geq \max _{1 \leq j \leq k}\left(\lceil(j-1) / 2\rceil+\left\lceil n_{j} / 2\right\rceil\right),
$$

where $n_{1} \geq n_{2} \geq \ldots \geq n_{k}$, as required.

Note that $\lceil(j-1) / 2\rceil=0$ when $j=1$. So the above inequality can be written as

$$
c\left(T_{S}^{\prime}, f\right) \geq \max \left\{\left\lceil n_{1} / 2\right\rceil, \quad \max 2 \leq j \leq k\left(\lceil(j-1) / 2\rceil+\left\lceil n_{j} / 2\right\rceil\right)\right\} .
$$

For the end section $T_{m}$, we have the same formula as Proposition 3.3. In order to get a uniform formula for intermediate sections and end sections (Propositions 3.2 and 3.3), we make a refinement of the decomposition of $T$ as follows. If $T_{1}$ is an iterated star $T_{S}^{\prime}=T^{\prime}\left(n_{1}, n_{2}, \ldots, n_{k}\right)$ with $n_{1} \geq n_{2} \geq \ldots \geq n_{k}$, and $u_{1}$ is the center of star $K_{1, n_{1}}$, which has as many hairs as possible, then we take $w_{0}=u_{1}$ and set $T_{0}$ to be the star $K_{1, n_{1}}$ induced by $\left\{w_{0}\right\} \cup N\left(w_{0}\right)$. Thus $T_{1}$ is decomposed into a star $T_{0}$ and an iterated star $T_{1}^{\prime}=T_{1}-u_{1}$. In this way, $T_{0}$ becomes an end section and $T_{1}^{\prime}$ becomes an intermediate section (we may denote it again by $T_{1}$ ). In the lower bound of Proposition 3.3, the first term $\left\lceil n_{1} / 2\right\rceil$ is for the end section $T_{0}$ and the second term $\max 2 \leq j \leq k\left(\lceil(j-1) / 2\rceil+\left\lceil n_{j} / 2\right\rceil\right)$ is for the intermediate section $T_{1}^{\prime}$, which agrees with the formula of Proposition 3.2.

Symmetrically, let $w_{m+1}$ be a vertex in $N\left(w_{m}\right) \backslash\left\{w_{m-1}\right\}$ with the largest number of hairs. Then we set $T_{m+1}$ to be a star induced by $\left\{w_{m+1}\right\} \cup N\left(w_{m+1}\right)$. Thus $T_{m}$ is decomposed into a star $T_{m+1}$ and an iterated star $T_{m}^{\prime}=T_{m}-w_{m+1}$.

In summary, we decompose $T$ into subtrees $T_{0}, T_{1}, \ldots, T_{m}, T_{m+1}$. Meanwhile, we may stipulate $p(T)=\left(w_{0}, w_{1}, w_{2}, \ldots, w_{m}, w_{m+1}\right)$ to be the backbone of $T$. In this context, $T_{0}$ and $T_{m+1}$ are stars, whose cutwidth values are given in Proposition 2.1; 


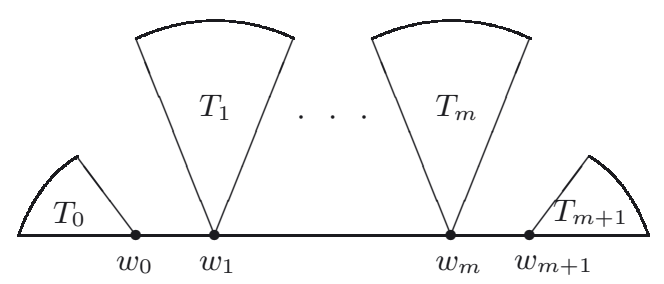

FiguRE 6. Further decomposition of iterated caterpillar.

and $T_{1}, \ldots, T_{m}$ are iterated stars, whose lower bounds are given in Proposition 3.2. This decomposition is shown in Figure 6.

For this decomposition, Proposition 2.3 can be written as:

$$
c(T) \geq \max _{0 \leq i \leq m+1} c\left(T_{i}\right) .
$$

\section{The MAIn Result}

With the foregoing preparation, we come to the formula of $c(T)$ for any iterated caterpillars $T$.

Theorem 4.1. For any iterated caterpillar $T=\cup_{i=0}^{m+1} T_{i}$, where $T_{0}$ and $T_{m+1}$ are stars and $T_{i}$ are iterated stars for $i=1, \ldots, m$, the cutwidth of $T$ is

$$
c(T)=\max _{0 \leq i \leq m+1} c\left(T_{i}\right),
$$

where $c\left(T_{0}\right)=\left\lceil\left|V\left(T_{0}\right)\right| / 2\right\rceil, c\left(T_{m+1}\right)=\left\lceil\left|V\left(T_{m+1}\right)\right| / 2\right\rceil$, and for $1 \leq i \leq m$, if $T_{i}$ is an iterated star $T\left(n_{1}, n_{2}, \ldots, n_{k}\right)$, then

$$
c\left(T_{i}\right)=\max _{1 \leq j \leq k}\left(\lceil j / 2\rceil+\left\lceil n_{j} / 2\right\rceil\right),
$$

where $n_{1} \geq n_{2} \geq \ldots \geq n_{k}$.

Proof. The lower bound given in the right-hand side of the above formula is due to Propositions 2.1, 2.3, and 3.2. We next show that this lower bound is attainable, namely, there exists a labeling $f^{*}$ such that $c\left(T, f^{*}\right)$ equals this lower bound, and thus $f^{*}$ is an optimal labeling and the lower bound is the minimum value. In fact, we can construct a labeling $f^{*}$ performing successively in the order of $T_{0}, T_{1}, T_{2}, \ldots, T_{m}, T_{m+1}$ such that when $f^{*}$ is restricted in each subtree $T_{i}$, it is optimal. In more detail, $f^{*}$ is constructed as follows.

Step 1. For $T_{0}=K_{1, n_{0}}$ with center $w_{0}$, we label the vertices by $\mathcal{N}_{0}=$ $\left\{1,2, \ldots, n_{0}+1\right\}$ such that $f\left(w_{0}\right)$ is a median of the label set $\mathcal{N}_{0}$. Set $\bar{n}:=n_{0}+1$ and $i:=1$. 


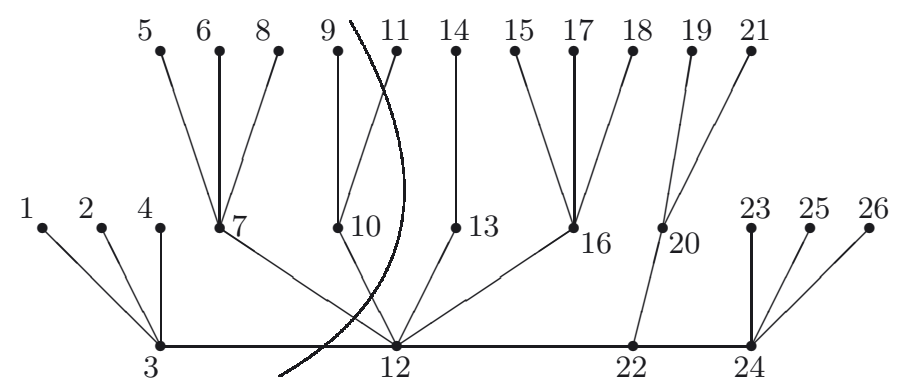

FiguRE 7. A tree with optimal labeling.

Step 2. For $T_{i}$ which is an iterated star $T\left(n_{1}, n_{2}, \ldots, n_{k}\right)$ (excluding the backbone leaves $\left.w_{i-1}, w_{i+1}\right)$ with vertex number $n\left(T_{i}\right)=n_{1}+n_{2}+\ldots+n_{k}+1$, we label the vertices by $\mathcal{N}_{i}=\left\{\bar{n}+1, \bar{n}+2, \ldots, \bar{n}+n\left(T_{i}\right)\right\}$ as follows.

By the notation of the previous section, suppose that $T^{1}=K_{1, n_{1}}, T^{2}=$ $K_{1, n_{2}}, \ldots, T^{k}=K_{1, n_{k}}$ are $k$ stars with centers $u_{1}, u_{2}, \ldots, u_{k}$ respectively, where $n_{1} \geq n_{2} \geq \ldots \geq n_{k}$. Moreover, let $\hat{T}^{j}=T^{j}-\{w\}$ for $j=1,2, \ldots, k$. We arrange them in the order $\hat{T}^{1}, \hat{T}^{3}, \ldots, \hat{T}^{k-1},\{w\}, \hat{T}^{k}, \ldots, \hat{T}^{4}, \hat{T}^{2}$ (if $k$ is even) or $\hat{T}^{1}, \hat{T}^{3}, \ldots, \hat{T}^{k},\{w\}, \hat{T}^{k-1}, \ldots, \hat{T}^{4}, \hat{T}^{2}$ (if $k$ is odd). Then we label them in turn by the consecutive numbers of $\mathcal{N}_{i}$ and in each subtree $T^{j}$ we set $f\left(u_{j}\right)$ to be a median of the label set $\left\{f(v): v \in V\left(T^{j}\right)\right\}$. Set $\bar{n}:=\bar{n}+n\left(T_{i}\right)$. If $i=m$, then go to the next step, else set $i:=i+1$ and go back to the beginning of this step.

Step 3. For $T_{m+1}=K_{1, n_{m+1}}$ with center $w_{m+1}$, we label the vertices by $\mathcal{N}_{m+1}=$ $\left\{\bar{n}+1, \bar{n}+2, \ldots, \bar{n}+n_{m+1}+1\right\}$ such that $f\left(w_{m+1}\right)$ is a median of the label set. Finally return the labeling $f^{*}$.

By Proposition 2.1 for stars, we have $c\left(T_{0}, f^{*}\right)=\left\lceil n_{0} / 2\right\rceil, c\left(T_{m+1}, f^{*}\right)=$ $\left\lceil n_{m+1} / 2\right\rceil$.

For $1 \leq i \leq m$, suppose that $T_{i}$ is an iterated star $T_{S}=T\left(n_{1}, n_{2}, \ldots, n_{k}\right)$ and $n_{1} \geq n_{2} \geq \ldots \geq n_{k}$. By viewing the proof of Proposition 3.2, we can see that $c\left(T^{j}, f^{*}\right)=\max \left\{\left|\partial\left(S_{f^{*}\left(u_{j}\right)-1}\right)\right|,\left|\partial\left(S_{f^{*}\left(u_{j}\right)}\right)\right|\right\}=\lceil j / 2\rceil+\left\lceil n_{j} / 2\right\rceil$. Hence $c\left(T_{S}, f^{*}\right)=\max _{1 \leq j \leq k} c\left(T^{j}, f^{*}\right)=\max _{1 \leq j \leq k}\left(\lceil j / 2\rceil+\left\lceil n_{j} / 2\right\rceil\right)$.

Therefore the labeling $f^{*}$ constructed by the above algorithm attains the lower

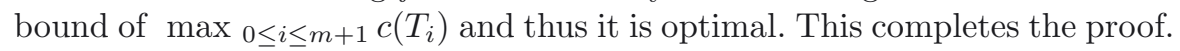

An example of optimal labeling is shown in Figure 7. The lower bound is given by an intermediate section $T(4,4,3,2)$, that is, $c\left(T_{S}, f\right) \geq \max _{1 \leq j \leq k}(\lceil j / 2\rceil+$ $\left.\left\lceil n_{j} / 2\right\rceil\right)=\max \{1+\lceil 4 / 2\rceil, 1+\lceil 4 / 2\rceil, 2+\lceil 3 / 2\rceil, 2+\lceil 2 / 2\rceil\}=4$. On the other hand, the labeling in Figure 7 has maximum size of coboundary $\left|\partial\left(S_{10}\right)\right|=4$, attaining the above lower bound. Therefore, this is an optimal labeling. 


\section{Concluding Remarks}

In the above discussion, we obtain the exact representations of cutwidth $c(T)$ for caterpillars and iterated caterpillars. Note that when all $n_{i}=2$, the formula of Theorem 4.1 for iterated caterpillars reduces to the formula of Proposition 2.2 for caterpillars. It is known that [15] presented an $O(n \log n)$ algorithm for computing the cutwidth of general trees. If we use the formula of Theorem 4.1 to compute the cutwidth of iterated caterpillars, the worst-case complexity is also $O(n \log n)$. However, the computation based on the formula is more straightforward.

The diameter of a tree may be called the length of the tree. Relatively, the width of a tree means the maximum distance from any leaf to the diameter path. Now, we determine the cutwidth value for trees with width 1 and 2. More classes of special graphs remain to be studied. For example, we may consider the doubly iterated caterpillars, each of which is a tree which yields an iterated caterpillar when all its leaves are removed, namely, the trees with width 3.

The cutwidth and the bandwidth of a graph are two closely related parameters in the VLSI designs, which are to minimize the congestion and the dilation respectively. In more detail, for a given labeling $f$ of $G$, the bandwidth of $f$ for $G$ is

$$
B(G, f)=\max \{|f(u)-f(v)|: u v \in E(G)\},
$$

which represents the maximum length of edges (dilation) in the embedding. The bandwidth of $G$ is $B(G)=\min \{B(G, f): f$ is a labeling of $G\}$.

The bandwidth problem for caterpillars is solved by Syslo and Zak [13]. Their result is as follows. Let $T$ be a caterpillar with backbone $p(T)=\left(w_{1}, w_{2}, \ldots, w_{m}\right)$. Denote by $T_{i j}$ the subtree of $T$ induced by $w_{i}, w_{i+1}, \ldots, w_{j-1}, w_{j}$ and all their neighbors. Then the bandwidth is $B(T)=\max _{i \leq j}\left\lceil\frac{\left|V\left(T_{i j}\right)\right|-1}{j-i+2}\right\rceil$. Moreover, a tree is called subdivided caterpillar if it is obtained from a caterpillar by subdividing the edges. Here, a subdivided edge is called a hair of length $k$ if the edge is replaced by a path of length $k$. Surprisingly, Monien [11] proves that the bandwidth problem for subdivided caterpillars with hair length 3 is NP-complete. Fortunately, the cutwidth problem for subdivided caterpillars is easier. In fact, by Lemma 1.1, if $T^{\prime}$ is a subdivided caterpillar obtained from a caterpillar $T$, then $c\left(T^{\prime}\right)=c(T)$. So the cutwidth problem for subdivided caterpillars is as easy as that for caterpillars, which is polynomially solvable (as seen in Prop. 2.2).

With respect to the relation of cutwidth and bandwidth, it can be seen that $c(T) \leq B(T)$ if $T$ is a star, caterpillar, or iterated caterpillar. The result for complete binary trees in $[2,7]$ also supports this inequality. So, it is interesting to characterize the graphs (or trees) $G$ with $c(G) \leq B(G)$ or $c(G) \geq B(G)$.

Acknowledgements. The authors would like to thank the referees for their helpful comments on improving the representation of the paper. 


\section{REFERENCES}

[1] J.A. Bondy and U.S.R. Murty, Graph Theory. Springer-Verlag, Berlin (2008).

[2] F.R.K. Chung, Labelings of graphs, edited by L.W. Beineke and R.J. Wilson. Selected Topics in Graph Theory 3 (1988) 151-168.

[3] F.R.K. Chung, On the cutwidth and topological bandwidth of a tree. SIAM J. Algbr. Discrete Methods 6 (1985) 268-277.

[4] J. Diaz, J. Petit and M. Serna, A survey of graph layout problems. ACM Comput. Surveys 34 (2002) 313-356.

[5] J.A. Gallian, A survey: recent results, conjectures, and open problems in labeling graphs. J. Graph Theory 13 (1989) 491-504.

[6] M.R. Garey and D.S. Johnson, Computers and Intractability: A Guide to the Theory of NP-Completeness. W.H. Freeman, San Francisco (1979).

[7] T. Lengauer, Upper and lower bounds for the min-cut linear arrangement of trees. SIAM J. Algbr. Discrete Methods 3 (1982) 99-113.

[8] Y. Lin, X. Li and A. Yang, A degree sequence method for the cutwidth problem of graphs. Appl. Math. J. Chinese Univ. Ser. B 17(2) (2002) 125-134.

[9] Y. Lin, The cutwidth of trees with diameter at most 4. Appl. Math. J. Chinese Univ. Ser. B 18(3) (2003) 361-368.

[10] H. Liu and J. Yuan, The cutwidth problem for graphs. Appl. Math. J. Chinese Univ. Ser. A 10(3) (1995) 339-348.

[11] B. Monien, The bandwidth minimization problem for caterpipals with hair length 3 is NP-complete. SIAM J. Algbr. Discrete Methods 7 (1986) 505-512.

[12] J. Rolin, O. Sykora and I. Vrt'o, Optimal cutwidths and bisection widths of 2- and 3dimensional meshes. Lect. Notes Comput. Sci. 1017 (1995) 252-264.

[13] M. M. Syslo and J. Zak, The bandwidth problem: critical subgraphs and the solution for caterpillars. Annal. Discrete Math. 16 (1982) 281-286.

[14] I. Vrt'o, Cutwidth of the r-dimensional mesh of d-ary trees. RAIRO Theor. Inform. Appl. 34 (2000) 515-519.

[15] M. Yanakakis, A polynomial algorithm for the min-cut arrangement of trees. J. ACM 32 (1985) 950-989.

Communicated by C. De Figueiredo.

Received September 7, 2012. Accepted December 19, 2012. 